\title{
HER4 promotes the progression of colorectal cancer by promoting epithelial-mesenchymal transition
}

\author{
XIAOJING JIA ${ }^{1}$, HUIEN WANG ${ }^{2}$, ZHONGXIN LI $^{3}$, JING YAN $^{1}$, YAN GUO ${ }^{4}$, \\ WUJIE ZHAO $^{1}$, LIXIA GAO ${ }^{1}$, BIN WANG $^{1}$ and YITAO JIA ${ }^{1}$ \\ Departments of ${ }^{1}$ Oncology and ${ }^{2}$ Thoracic Surgery, Hebei General Hospital, Shijiazhuang, Hebei 050051; \\ ${ }^{3}$ Second Department of Surgery, The Fourth Hospital of Hebei Medical University; ${ }^{4}$ Fifth Department of Oncology, \\ The First Hospital of Shijiazhuang, Shijiazhuang, Hebei 050011, P.R. China
}

Received January 30, 2019; Accepted January 6, 2020

DOI: $10.3892 / \mathrm{mmr} .2020 .10974$

\begin{abstract}
Colorectal cancer (CRC) remains one of the most common cancer types worldwide. A few previous studies have examined whether HER4 may promote the progression of CRC. The present study examined the associations among the expression levels of members of the HER family, and investigated the potential mechanism underlying the function of HER4 in CRC cells. Immunohistochemistry analysis was conducted to detect the expression levels of HER family members in patients with CRC. HER4 expression was knocked down using short hairpin RNA in HCT116 cells, and confirmed by quantitative PCR and western blotting. The proliferation and adhesion of CRC cells were analyzed by CCK-8 assays and adhesive assays, respectively. Flow cytometry was used to measure cell apoptosis. Western blotting and immunofluorescence staining in CRC cells were performed to identify proteins related to epithelial-mesenchymal transition. The proportion of patients with CRC presenting positive expression of the HER family members epidermal growth factor receptor (EGFR), HER2, HER3 and HER4 were 72.1, 45.2, 43.8 and $34.2 \%$, respectively. No relationship was found between HER4 and EGFR, HER2 or HER3 expression. Higher expression of HER4 was positively associated with lymph node metastasis $(\mathrm{P}=0.039)$. In the present study, HER4 expression was found to be associated with an unfavorable clinical outcome in patients with CRC $\left(\mathrm{P}_{\text {logrank }}=0.020\right)$. Cell proliferation was inhibited, and apoptosis was increased following HER4 knockdown. Furthermore, HER4 knockdown increased the expression of E-cadherin and decreased the expressions of $\mathrm{N}$-cadherin and vimentin $(\mathrm{P}<0.05)$. HER4 expression was found to be unrelated to other HER family members. In the present study,
\end{abstract}

Correspondence to: Professor Yitao Jia, Department of Oncology, Hebei General Hospital, 348 Heping Road, Shijiazhuang, Hebei 050051, P.R. China

E-mail: jiayitao99@163.com

Key words: colorectal cancer, HER4, epithelial-mesenchymal transition, metastasis, prognosis positive expression of HER4 promoted the progression of CRC through epithelial-mesenchymal transition.

\section{Introduction}

Colorectal cancer (CRC) is one of the most common cancer types worldwide, being the third most frequently diagnosed malignancy in males and second most in females (1). Currently, radical surgical resection is the most effective treatment for CRC; however, most patients, in particular elderly patients, suffer from high local recurrence and distant metastasis $(2,3)$. Therefore, it is important to identify prognostic markers to facilitate the clinical treatment of CRC.

The HER family, which encodes for tyrosine kinase receptors, includes four members, HER1 [also known as epidermal growth factor receptor (EGFR)], HER2, HER3 and HER4 (4). Previous studies have shown the relationship between the survival of patients and the expression of HER2, HER3 and EGFR (5-7). Rego et al (5) reported that EGFR overexpression was detected in $80-90 \%$ of CRC, and was associated with poor disease-free survival and overall survival. Kapitanović et al (6) showed that HER2 expression was correlated with the stage of disease and survival in CRC. Mitsui et al (7) reported that the expression of HER3 led to metastases in the lymph node and liver, and poorer patient prognosis in CRC. However, to the best of our knowledge, few studies have investigated whether HER4 may serve a role in the process of epithelial-mesenchymal transition (EMT) in CRC. Therefore, the present study examined the role of HER4 in CRC. Previous studies have shown that HER4 has four receptor isoforms generated by alternative splicing, extracellular juxtamembrane domains (JM-a and JM-b) and cytoplasmic domains (CYT-1 and CYT-2) (8). HER4 regulates cell proliferation, survival and differentiation, and is expressed both in fetal and normal tissues (4). An increasing number of studies have demonstrated that HER4 may be involved in tumorigenesis (9-11). Nielsen et al (10) found that HER4 expression is associated with short progression-free survival in malignant melanoma. Wang et al (11) showed that HER4 expression promotes osteosarcoma cell proliferation and tumorigenesis, and reduced apoptosis both in vitro and in vivo. In our previous study, CYT-2, but not CYT-1, could significantly promote CRC progression (12). 
EMT initiates the process of metastasis in tumor progression (13-15). Through EMT, tumor cells often lose their epithelial cell phenotype, downregulating E-cadherin expression, and acquire a mesenchymal cell phenotype, expressing $\mathrm{N}$-cadherin and Vimentin. Vu et al (16) identified that the progression of EMT is regulated by $\mathrm{WNT} / \beta$-catenin and that EMT transcription factors affect the metastasis of CRC. However, to the best of our knowledge, few studies have evaluated whether HER4 may serve a role in the process of EMT in CRC.

The present study investigated the expression of HER family members in patients with CRC and the effects of HER4 on CRC cell proliferation, survival, migration and EMT.

\section{Materials and methods}

Patients and tumor specimens. All procedures involving human participants were approved by the ethical standards of the institutional research committee of The Fourth Hospital of Hebei Medical University and according to the 1964 Helsinki Declaration and its later amendments or comparable ethical standards. The participants signed an informed consent form after being informed about the benefits and risks of the procedure in this study. In total, 73 paraffin-embedded primary colorectal cancer specimens were collected from the Department of Surgery, Fourth Hospital of Hebei Medical University, from January 2008 to February 2012. All patients (36 males and 37 females, 24-83 years old) were confirmed to have adenocarcinoma, and no patient had been administered chemotherapy or radiation therapy before surgery.

Immunohistochemistry (IHC) and antibodies. IHC staining was performed using tissues (thickness, $5 \mu \mathrm{m}$ ) from patients with CRC after surgery. The tissues were fixed with $10 \%$ formalin at room temperature for $24 \mathrm{~h}$ and embedded in paraffin at $62^{\circ} \mathrm{C}$ for $45 \mathrm{~min}$. The sections were first incubated with $10 \%$ normal goat serum for $10 \mathrm{~min}$ at $37^{\circ} \mathrm{C}$ to block nonspecific immunoglobulin binding. Then the sections were incubated with anti-EGFR antibody (1:50; cat. no. GTX121919; Genetex, Inc.), anti-Her2 antibody (1:100; cat. no. GTX117480; Genetex, Inc.), anti-c-HER3 antibody (1:100; cat. no. ARG52855; Arigo Biolaboratories Corp.), and anti-c-HER4 antibody (1:50; cat. no. ARG52859; Arigo Biolaboratories Corp.) overnight at $4^{\circ} \mathrm{C}$. The sections were washed with PBS buffer. Secondary antibodies (Rabbit SP kit; cat. no. SP-9000; OriGene Technologies, Inc.) were added to the tissue sections and incubated at $37^{\circ} \mathrm{C}$ for $30 \mathrm{~min}$. All procedures were conducted according to the manufacturer's instructions provided with the Rabbit SP Kit (OriGene Technologies, Inc.). All tumor slides were examined under a light microscope.

According to the scoring system approved by the US Food and Drug Administration (17), samples were scored semi-quantitatively for EGFR, HER2, HER3 and HER4 staining as follows: i) 0 , no immunostaining or staining in $<10 \%$ of CRC cells, ii) $1+$, incomplete staining of $>10 \%$ of CRC cells, iii) $2+$, weak-to-moderately complete staining of $>10 \%$ of CRC cells and iv) $3+$, moderate-to-strongly complete staining of $>10 \%$ of CRC cells. Scores of 0 or $1+$ were regarded as negative for EGFR, HER2, HER3 and HER4 expression, while scores of $2+$ and $3+$ were regarded as positive for EGFR, HER2, HER3 and HER4 expression.

HER4 knockdown cell lines and cell culture. In total, four hairpin RNAs were constructed to specifically target HER4 mRNA by using short hairpin RNA (shRNA) design tools, GenBank (https://www.ncbi.nlm.nih.gov/genbank/). Using Basic Local Alignment Search Tool (BLAST+ 2.3.0; http://blast.ncbi.nlm.nih.Gov/Blast.cgi), only the selected gene was targeted by the designed shRNAs. The sequences of the four designed shRNAs are shown in Table I. HER4 was previously reported to be overexpressed in the HCT116 CRC cell line (18); therefore, this cell line was used in the present study. The lentivirus (Shanghai GenePharma, Co., Ltd.) was infected (400 $\mu \mathrm{l}$ lentiviral fluid per $2 \mathrm{ml}$ medium) with HCT116 CRC cells by polybrene (cat. no. H9268; Sigma-Aldrich; Merck KGaA). The expression levels of HER4 were determined by quantitative PCR (qPCR) and western blotting (shown in Figs. S1 and S2). qPCR was performed by FTC-3000 (Funglyn Biotech, Inc.), SYBR Real-Time PCR kit (cat. no. E22001; Shanghai GenePharma, Co., Ltd.) was used according to the manufacture's protocols. qPCR thermocycling conditions were: $95^{\circ} \mathrm{C}$ for $3 \mathrm{~min} ; 40$ cycles were performed at $95^{\circ} \mathrm{C}$ for $30 \mathrm{sec}$ and $62^{\circ} \mathrm{C}$ for $40 \mathrm{sec}$. The primer sequences used were as follows: HER4 forward, 5'-CCGAGGATGAGTATGTGAATGA-3' and reverse, 5'-AGGTGGCAGGCTGTGGTT-3'; $\beta$-actin forward, 5'-CGTGGACATCCGCAAAGA and reverse, 5'-GAAGGT GGACAGCGAGGC-3'. Sense and antisense sequences of the stem-loop of shRNA HER4-4 and nc-shRNA are shown in Table II. All the procedures above were performed without any treatment. Three groups were evaluated in the experiment: i) HCT116 group; ii) negative control (nc-shRNA) group; and iii) silenced HER4 (shRNA-HER4) group.

The human CRC cell line HCT116 was obtained from the Type Culture Collection of the Chinese Academy of Sciences. Cells were cultured for 24-48 h in McCoy's 5A (cat. no. 16600082; Gibco; Thermo Fisher Scientific, Inc.) medium supplemented with 10\% FBS (cat. no. 10099141; Gibco; Thermo Fisher Scientific, Inc.) and penicillin $(100 \mathrm{U} / \mathrm{ml})$-streptomycin $(100 \mu \mathrm{g} / \mathrm{ml})$ at $37^{\circ} \mathrm{C}$ in $5 \% \mathrm{CO}_{2}$.

Cell proliferation assay. Cell proliferation was measured using a CCK-8 (Dojindo Molecular Technologies, Inc.), according to the manufacturer's protocols (19). Cells were seeded into 96-well plates at a density of $3 \times 10^{3}$ cells $/ 100 \mu \mathrm{l}$ and incubated at $37^{\circ} \mathrm{C}$ in $5 \% \mathrm{CO}_{2}$ for 24,48 and $72 \mathrm{~h}$. Cells were incubated with $10 \mu \mathrm{l} \mathrm{CCK}-8$ reagent for another $2 \mathrm{~h}$. The optical density (OD) at $450 \mathrm{~nm}$ of cells was measured using the BioTek elx800 (BioTek Instruments, Inc.).

Flow cytometry assay. To detect the apoptosis of CRC cells, cells were stained with FITC and propidium iodide (PI) at room temperature for $15 \mathrm{~min}$ in the dark. A cell apoptosis assay was performed by flow cytometry (BD FACSVerse ${ }^{\mathrm{TM}}$; BD Biosciences), and the flow cytometry data were analyzed using BD FACSuite ${ }^{\mathrm{TM}}$ Software (BD Biosciences).

Cell adhesive assay. To evaluate adhesive ability of CRC cells, $6 \times 10^{4}$ cells/well were seeded into six-well plates containing Matrigel (cat. no. 356234, Corning, Inc.) and incubated at $37^{\circ} \mathrm{C}$ 
Table I. Sequences of shRNAs targeting HER4.

\begin{tabular}{lcc}
\hline Number & Name & Sequences (5'-3') \\
\hline shRNA HER4-1 & HER4-homo-1105 & GCATTGGCACAGGATCATTGA \\
shRNA HER4-2 & HER4-homo-1815 & GGTCCTGACAACTGTACAAAG \\
shRNA HER4-3 & HER4-homo-2078 & GCTCTTCATTCTGGTCATTGT \\
shRNA HER4-4 & HER4-homo-2760 & GCTCTGGAGTGTATACATTAC
\end{tabular}

shRNA, short hairpin RNA.

Table II. Sense and antisense sequences of the stem-loop of shRNA-HER4-4 and nc-shRNA.

\begin{tabular}{ll}
\hline Name & \multicolumn{1}{c}{ Sequences } \\
\hline shRNA-HER4-4 & Sense: 5'-GATCCGCTCTGGAGTGTATACATTACTTCAAGAGAGTAATGTATACACTCCAGAGCTT \\
& TTTTG-3' \\
& Antisense: 5'-AATTCAAAAAAGCTCTGGAGTGTATACATTACTCTCTTGAAGTAATGTATACACTC \\
& CCAGAGCG-3' \\
nc-shRNA & Sense: 5'-GATCCGTTCTCCGAACGTGTCACGTTTCAAGAGAACGTGACACGTTCGGAGAACTT \\
& TTTTG-3' \\
& Antisense: 5'-AATTCAAAAAAGTTCTCCGAACGTGTCACGTTCTCTTGAAACGTGACACGTTCG \\
& GAGAACG-3' \\
\hline
\end{tabular}

shRNA, short hairpin RNA; nc-shRNA, negative control short hairpin RNA.

in $5 \% \mathrm{CO}_{2}$ for 1 or $2 \mathrm{~h}$, and then washed with PBS three times. The OD of cells was determined using the BioTek elx800. The wavelength of measurement was $490 \mathrm{~nm}$.

Wound healing assay. In total, $5 \times 10^{6}$ cells/well were seeded in six-well plates. A scratch was made with a $200-\mu$ l tip on the monolayer of CRC cells. Then, cells were washed with PBS to remove detached cells. CRC cells were cultured in MyCoy's 5A medium (cat. no. 16600082; Gibco; Thermo Fisher Scientific, Inc.) with 2\% FBS (cat. no. 10099141; Gibco; Thermo Fisher Scientific, Inc.) and $1 \%$ penicillin-streptomycin in $37^{\circ} \mathrm{C}$. The images were captured using an inverted microscope (magnification, x 50) at 0, 12, 24 and $48 \mathrm{~h}$. Wound healing area was measured by calculating the wound area in each period. The area of the wound was calculated using Image J 1.49 p software (20), and calculated by the equation: The percentage of wound healing area $=[1$-(wound area at $\mathrm{Tt} /$ wound area at T0)] x100, where $\mathrm{Tt}$ is the time passed since wounding $(12,24$ and $48 \mathrm{~h}$ ) and T0 is the time of initial wounding.

Western blotting. Cells were lysed in RIPA buffer (cat. no. BB-3201; BestBio). BCA protein assay kit (cat. no. P0012S; Beyotime Institute of Biotechnology) was used for the determination of total protein concentration. A total of $40 \mu \mathrm{g}$ of protein lysate was separated by $9 \%$ SDS-PAGE and transferred onto a PVDF membrane (EMD Millipore). The membranes were blocked with $5 \%$ non-fat dry milk for $2 \mathrm{~h}$ at room temperature. The membranes were incubated with a primary antibody at $4^{\circ} \mathrm{C}$ overnight, followed by incubation with a secondary antibody (HRP-conjugated goat anti-Rabbit IgG; 1:2,000; cat. no. ZB2301; OriGene Technologies, Inc.) for $1 \mathrm{~h}$ at $37^{\circ} \mathrm{C}$. The protein signals were detected by DAB (OriGene Technologies, Inc.). Primary antibodies included, anti-E cadherin antibody (1:300; cat. no. PB0583; Wuhan Boster Biological Technology, Ltd.), anti-N cadherin antibody (1:200; cat. no. BM-1573; Wuhan Boster Biological Technology, Ltd.), vimentin (1:100; cat. no. BM0135; Wuhan Boster Biological Technology, Ltd.) and $\beta$-actin $(1: 1,000$, cat. no. TA-09; OriGene Technologies, Inc.). The intensity of bands were calculated using Tanon 1600 Gel Imaging system (Tanon Sciences and Technology Co., Ltd.).

Immunofluorescence double staining. CRC cells were fixed in 4\% paraformaldehyde for $15 \mathrm{~min}$ at room temperature. CRC cells were incubated with $10 \%$ normal goat serum (OriGene Technologies, Inc.) for $2 \mathrm{~h}$ at room temperature. Then, CRC cells were incubated with primary antibodies overnight at $4^{\circ} \mathrm{C}$. CRC cells were double-stained $(60 \mathrm{~min}$ at room temperature) with a mixture of primary antibodies against E-cadherin (1:100; cat. no. PB0583; Wuhan Boster Biological Technology, Ltd.) and Vimentin (1:50; cat. no. BM4029; Wuhan Boster Biological Technology, Ltd.), and cultured with a mixture of the fluorescent secondary antibodies FITC (1:75; cat. no. ZF-0311; goat anti-rabbit; OriGene Technologies, Inc.) and tetramethylrhodamine (1:90; cat. no. ZF-0313; goat anti-mouse; OriGene Technologies, Inc.). The cells were then washed with PBS and observed using a fluorescence-inverted microscope (magnification, x400). Image-Pro Plus 6.0 (Media Cybernetics, Inc.) was used to evaluate the densitometry. 

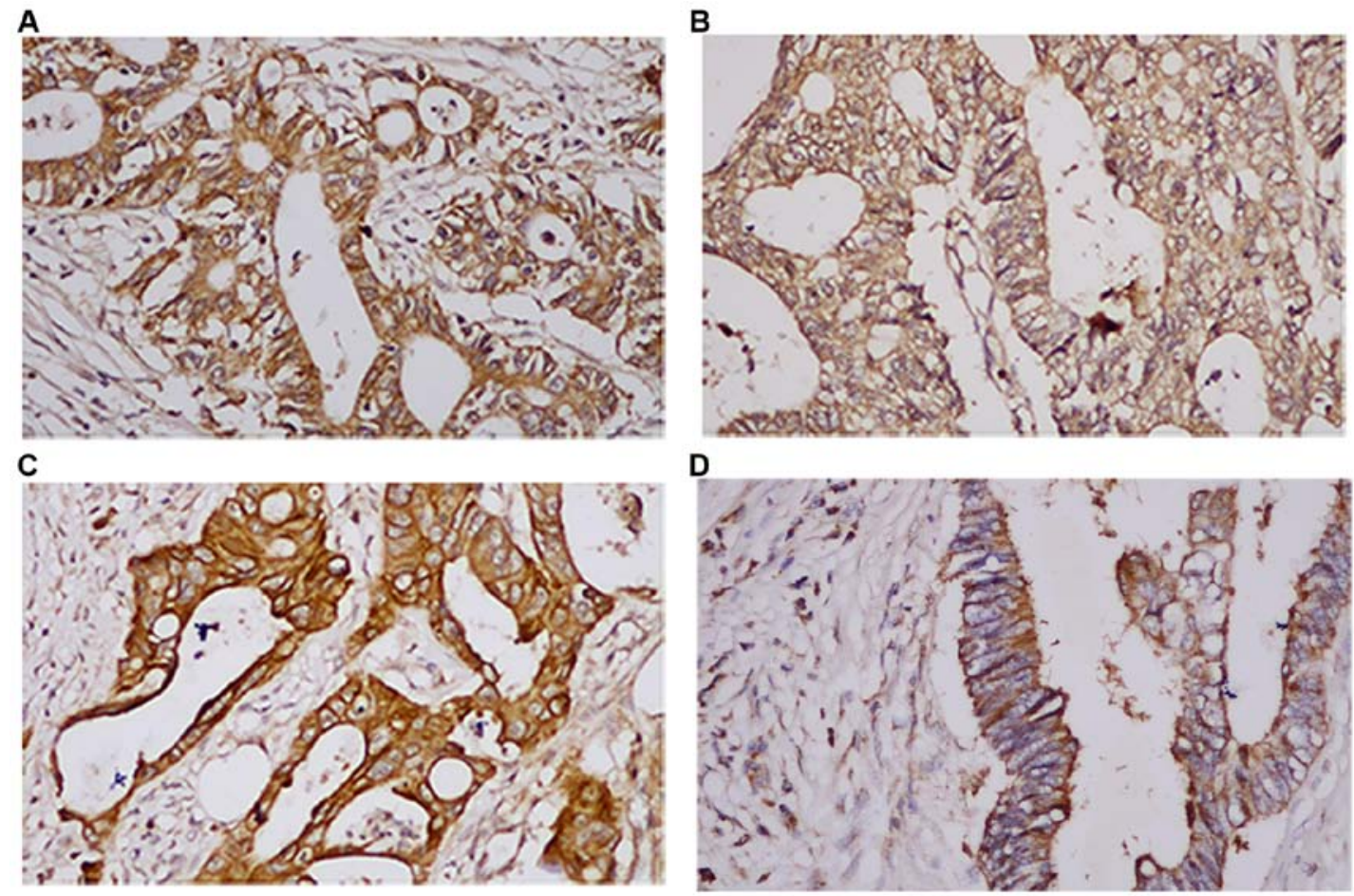

Figure 1. Immunohistochemical staining for EGFR, HER2, HER3 and HER4 in CRC. (A) Positive EGFR staining. (B) Positive HER2 staining. (C) Positive HER3 staining. (D) Positive HER4 staining. HER family members showed varying expression levels in CRC tissues. Magnification, x200. EGFR, epidermal growth factor receptor; $\mathrm{CRC}$, colorectal cancer.

Statistical analysis. Pearson's $\chi^{2}$ test was used to determine the correlations of clinicopathological parameters. All data are representative of $\geq 3$ independent experiments. Kaplan-Meier plots and log-rank tests were used to analyze 5-year overall survival rate. Student's t-test was used to detect differences between two groups, and one-way ANOVA was used to determine the differences among multiple groups followed by a Student-Newman-Keuls-q post hoc test. Kendall rank correlation coefficient was used to compare correlations among HER family members. Statistical analysis was performed using SPSS v.21.0 software (IBM Corp.). Data are presented as the mean $\pm \mathrm{SD}$. $\mathrm{P}<0.05$ was considered to indicate a statistically significant difference.

\section{Results}

Expression of HER family members in 73 patients with CRC. A total of 90 patients were recorded; however, 17 patients were not followed up. IHC was used to examine the expression of HER family members in primary tumor tissues. The positive expression rates of EGFR, HER2, HER3 and HER4 were 72.1, $45.2,43.8$ and $34.2 \%$, respectively (Fig. 1). The associations among EGFR, HER2, HER3 and HER4 are summarized in Table III. The present results suggested that HER4 expression was not associated with EGFR, HER2 or HER3 expression.

Positive expression of HER4 is positively associated with lymph node metastasis in patients with CRC. The present study investigated the relationship between HER4 expression and clinicopathological features, including gender, age, tumor site, T stage, TNM stage and lymph node metastasis (Table IV). The present results suggested that positive rates of HER4 expression were higher in patients with lymph node metastasis than those without lymph node metastasis (Table IV; 60.0 vs. $40.0 \%$; $P=0.039$ ). Additionally, staining experiments showed that HER4 can be found the in cell membrane, cytoplasm and nucleus of CRC (Fig. S3).

Positive expression of HER4 is associated with a poor 5-year survival rate. Kaplan-Meier survival analysis showed that the 5-year survival rate for patients with positive HER4 expression was $64.3 \%$, whilst for patients with negative HER4 expression the 5-year survival rate was $85.5 \%(\mathrm{P}=0.02)$. The present results suggested that the positive expression of HER4 indicated an unfavorable prognosis in patients with CRC (Fig. 2).

HER4 knockdown suppresses CRC cell proliferation. CCK-8 assay was used to determine the OD in HCT116 transfected with shRNA-HER4 and nc-shRNA, and untransfected. After $24 \mathrm{~h}$ the OD values were $0.552 \pm 0.006,0.497 \pm 0.040$ and $0.555 \pm 0.016$, respectively. At $48 \mathrm{~h}$, the OD values were $2.268 \pm 0.014,1.901 \pm 0.027$ and $2.221 \pm 0.057$. At $72 \mathrm{~h}$ the values were $2.875 \pm 0.031,2.276 \pm 0.021$ and $2.221 \pm 0.061$. The present results indicated that knockdown of HER4 significantly inhibited CRC cell proliferation (Fig. 3).

HER4 knockdown increases apoptosis and the adhesive ability of CRC cells. Flow cytometry showed that the rate of apoptosis in shRNA-HER4 was $3.43 \pm 0.67 \%$, which was significantly higher compared with the other experimental groups (Fig. 4; $\mathrm{P}<0.05$ ). The present results suggested that HER4 knockdown enhanced apoptosis in CRC cells.

Cell adhesion assays indicated that cell adhesion in cells transfected with shRNA-HER4 was enhanced compared to 
Table III. Correlation among the expression of EGFR, HER2, HER3 and HER4 in CRC $(n=73)$.

\begin{tabular}{cccc}
\hline & EGFR & HER2 & HER3 \\
\hline HER2 & $0.273(0.020)^{\mathrm{a}}$ & - & - \\
HER3 & $0.439(0.001)^{\mathrm{b}}$ & $0.362(0.002)^{\mathrm{b}}$ & - \\
HER4 & $-0.052(0.662)$ & $0.099(0.403)$ & $-0.056(0.636)$ \\
\hline
\end{tabular}

EGFR, epidermal growth factor receptor; CRC, colorectal cancer. Kendall's $\tau$ (Kendall rank correlation coefficient) was used to compare correlations among HER family members. ${ }^{\mathrm{a}} \mathrm{P}<0.05$, EGFR vs. HER2. ${ }^{\mathrm{b}} \mathrm{P}<0.01$, EGFR vs. HER3, and HER2 vs. HER3.

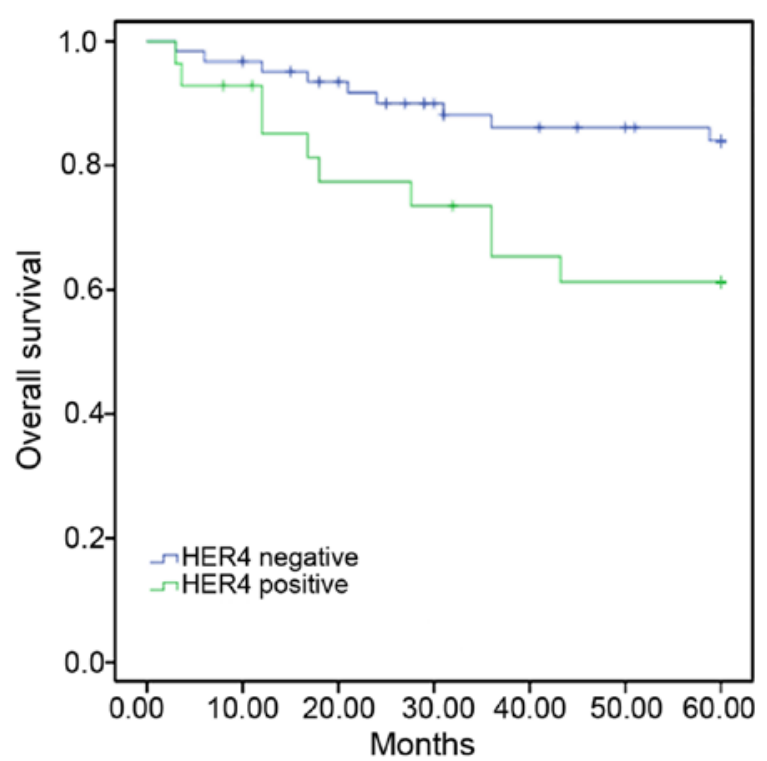

Figure 2. Kaplan-Meier analysis of OS stratified by level of HER4 expression in patients with CRC. HER4 negative expression was significantly associated with an extended OS compared with patients with positive HER4 expression. $\mathrm{P}=0.020$. OS, overall survival; $\mathrm{CRC}$, colorectal cancer.

that in the HCT116 and nc-shRNA groups (Fig. 5; P<0.05). A wound healing assay demonstrated that cell migration in shRNA-HER4 was inhibited compared with the other experimental groups (Fig. 6; $\mathrm{P}<0.05$ ). The present results suggested that HER4 increased cellular migration and inhibited adhesion activity, which may increase migration in malignant tumors.

HER4 knockdown inhibits the protein expression levels of EMT associated factors. Western blotting was performed to examine EMT-related proteins. The present study found that vimentin and $\mathrm{N}$-cadherin expression was decreased, while E-cadherin expression was increased after HER4 knockdown compared with the other experimental groups (Fig. 7). However, no significant differences were found between the HCT116 group and nc-shRNA group (Fig. 7). Immunofluorescent double-staining was conducted to identify and measure E-cadherin and vimentin expression in CRC cells. HER4 knockdown led to increased E-cadherin expression levels and decreased vimentin expression levels (Fig. 8).

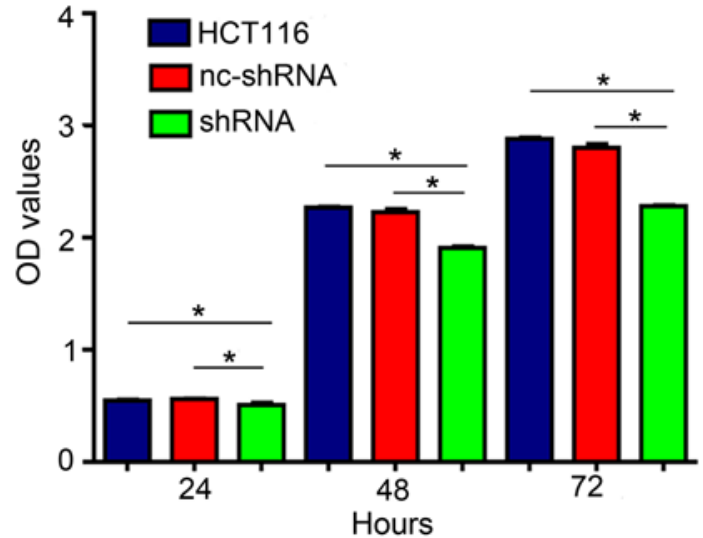

Figure 3. Knockdown of HER4 in colorectal cancer cells inhibits cell proliferation. OD values were evaluated at 24,48 and $72 \mathrm{~h}$. HCT116 represents the control group, nc-shRNA represents the negative group and shRNA represents the HER4 knockdown group. Data are presented as the mean \pm SD from three independent experiments. "P $<0.05$. OD, optical density; shRNA, short hairpin RNA; nc-shRNA, negative control short hairpin RNA.

These results suggested that knockdown of HER4 suppressed EMT in CRC cells.

\section{Discussion}

In the present study, although EGFR, HER2, HER3 and HER4 showed varying expression in CRC, positive expression of HER4 was not associated with EGFR, HER2 and HER3. The positive rate of HER4 expression was higher in patients with lymph node metastasis, suggesting that positive HER4 expression may indicate an unfavorable outcome in patients with CRC. The present analyses suggested that positive HER4 expression enhanced proliferation, reduced apoptosis and the adhesive ability of CRC cells, and promoted the expression of biomarkers considered to be associated with EMT.

In the present study, the positive expression rates of the HER family members EGFR, HER2, HER3 and HER4 were $72.1,45.2,43.8$ and $34.2 \%$, respectively. The present results are consistent with those of previous studies showing that the expression of HER family members in CRC varies widely $(7,18,21-23)$. These discrepancies can be explained by differences in the mouse model experiment of methodology, mouse model age, antibodies and subject ethnicity in the studies (21). Expression of HER1 is known to be closely associated with HER2 expression (24-27). In breast cancer, Smad3, which can convert transforming growth factor- $\beta$ into a carcinogenic factor, is activated when HER1 and HER 2 are co-expressed, resulting in tumor progression (24). The functional tyrosine domain of HER2 activates HER3 or HER1, promoting tumor development via activation of signaling pathways such as STAT3, RAS-mitogen-activated protein kinase and PI3K (25-27). Previous studies showed that the expression of HER1, HER2 and HER 3 exhibits complex interactions (24-27). The present results suggested that HER4 had no significant association with HER1, HER2 or HER3 in the tissue collected from patients with CRC. Although Lee et al (28) showed that co-expression of HER2 and HER4 resulted in a shorter prognosis in CRC, they did not observe a relationship between HER2 and HER4 expression; which is 
Table IV. Association between clinicopathological characteristics and HER4 expression in 73 patients with CRC.

\begin{tabular}{|c|c|c|c|c|c|}
\hline \multirow[b]{2}{*}{ Parameter } & \multirow[b]{2}{*}{ Number of patients (\%) } & \multicolumn{2}{|c|}{ HER4 expression in CRC } & \multirow[b]{2}{*}{$\chi^{2}$} & \multirow[b]{2}{*}{ P-value } \\
\hline & & Negative (\%) & Positive (\%) & & \\
\hline Sex & & & & 0.680 & 0.282 \\
\hline Male & $36(49.3)$ & $22(45.8)$ & $14(56.0)$ & & \\
\hline Female & $37(50.7)$ & $26(54.2)$ & $11(44.0)$ & & \\
\hline Age & & & & 0.00 & 0.594 \\
\hline$\leq 60$ years & $38(52.1)$ & $25(52.1)$ & $13(52.0)$ & & \\
\hline$>60$ years & $35(47.9)$ & $23(47.9)$ & $12(48.0)$ & & \\
\hline Tumor site & & & & 0.089 & 0.485 \\
\hline Colon & $45(61.6)$ & $29(60.4)$ & $16(64.0)$ & & \\
\hline Rectum & $28(38.4)$ & $19(39.6)$ & $9(36.0)$ & & \\
\hline T stage & & & & 0.978 & 0.807 \\
\hline $\mathrm{T}_{1}$ & $2(2.7)$ & $1 \quad(2.1)$ & $1(4.0)$ & & \\
\hline $\mathrm{T}_{2}$ & $14(19.2)$ & $10(20.8)$ & $4(16.0)$ & & \\
\hline $\mathrm{T}_{3}$ & $22(30.1)$ & $13(27.1)$ & $9(36.0)$ & & \\
\hline $\mathrm{T}_{4}$ & $35(47.9)$ & $24(50.0)$ & $11(44.0)$ & & \\
\hline TNM stage & & & & 1.029 & 0.222 \\
\hline $\mathrm{I}+\mathrm{II}$ & $41(56.2)$ & $29(60.4)$ & $12(48.0)$ & & \\
\hline III & $32(43.8)$ & $19(39.6)$ & $13(52.0)$ & & \\
\hline Lymph node metastasis & & & & 4.035 & $0.039^{\mathrm{a}}$ \\
\hline Positive & $41(56.2)$ & $31(64.6)$ & $10(40.0)$ & & \\
\hline Negative & $32(43.8)$ & $17(35.4)$ & $15(60.0)$ & & \\
\hline
\end{tabular}

CRC, colorectal cancer. Chi-square tests were used to compare parameters between positive and negative groups for HER 4 expression. ${ }^{a} \mathrm{P}<0.05$.
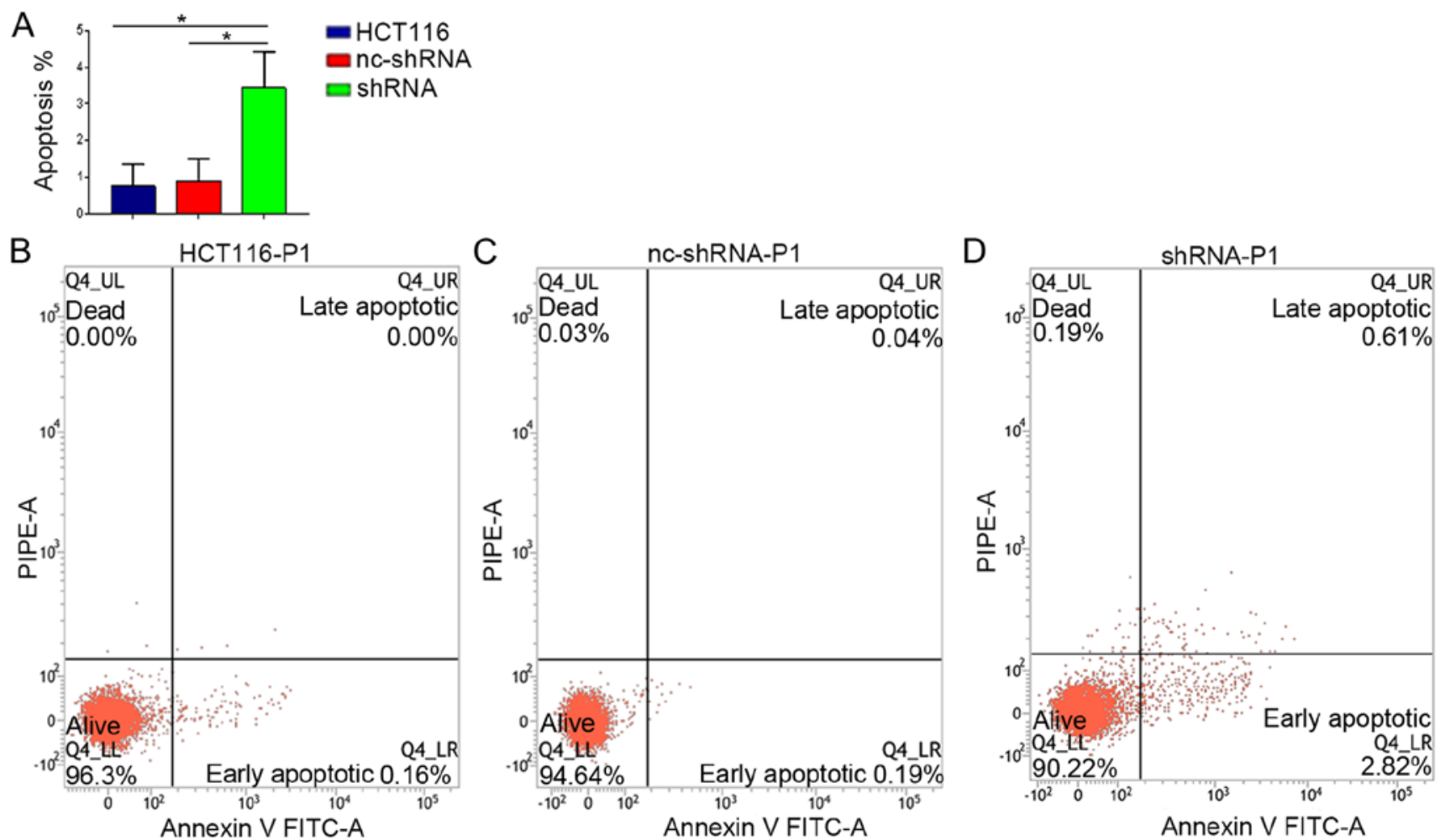

Figure 4. Knockdown of HER4 increases apoptosis in CRC cells. (A) Quantification of apoptosis. Results of flow cytometry assays for (B) HCT116, (C) nc-shRNA and (D) shRNA. Data are presented as the mean \pm SD from three independent experiments. "P $<0.05$. CRC, colorectal cancer; shRNA, short hairpin RNA; nc-shRNA, negative control short hairpin RNA. 


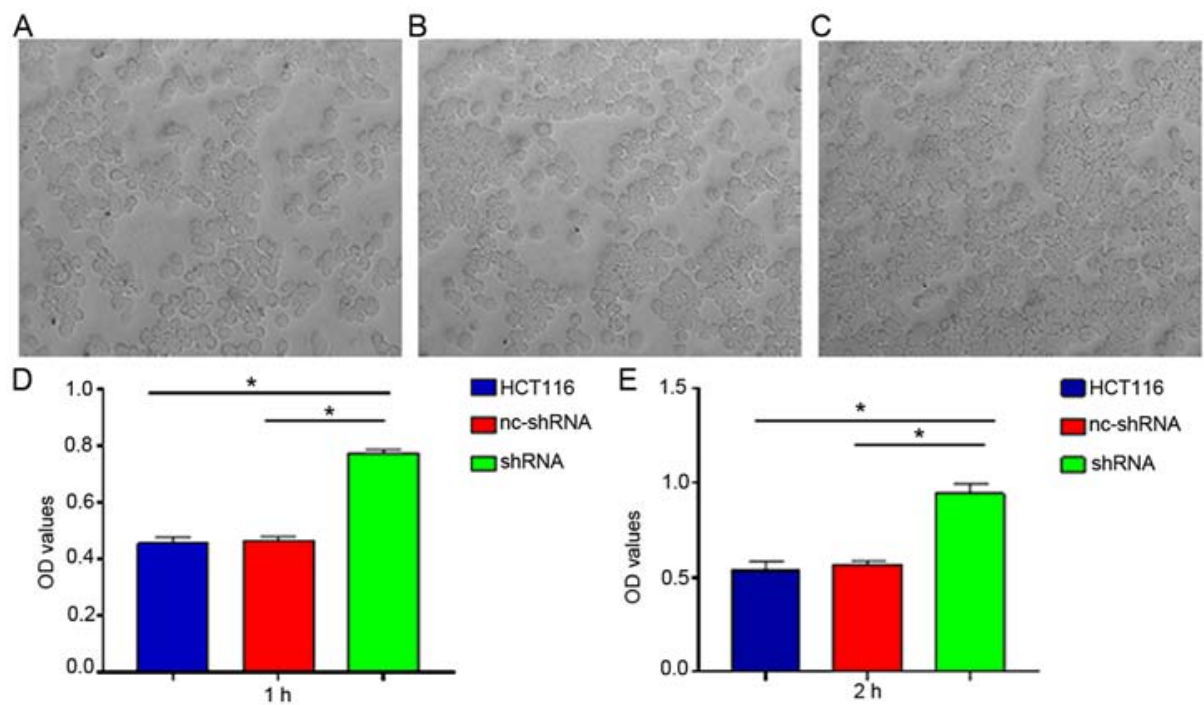

Figure 5. Knockdown of HER4 inhibits the adhesive ability of CRC cells. OD values were determined at 1 and $2 \mathrm{~h}$. Adhesion assay results in (A) HCT116, (B) nc-shRNA and (C) shRNA. OD values of the three groups at (D) 1 and (E) $2 \mathrm{~h}$. Data are presented as the mean \pm SD of three independent experiments. "P<0.05. CRC, colorectal cancer; OD, optical density; shRNA, short hairpin RNA; nc-shRNA, negative control short hairpin RNA.

A

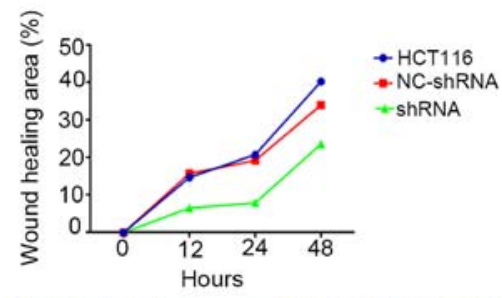

B

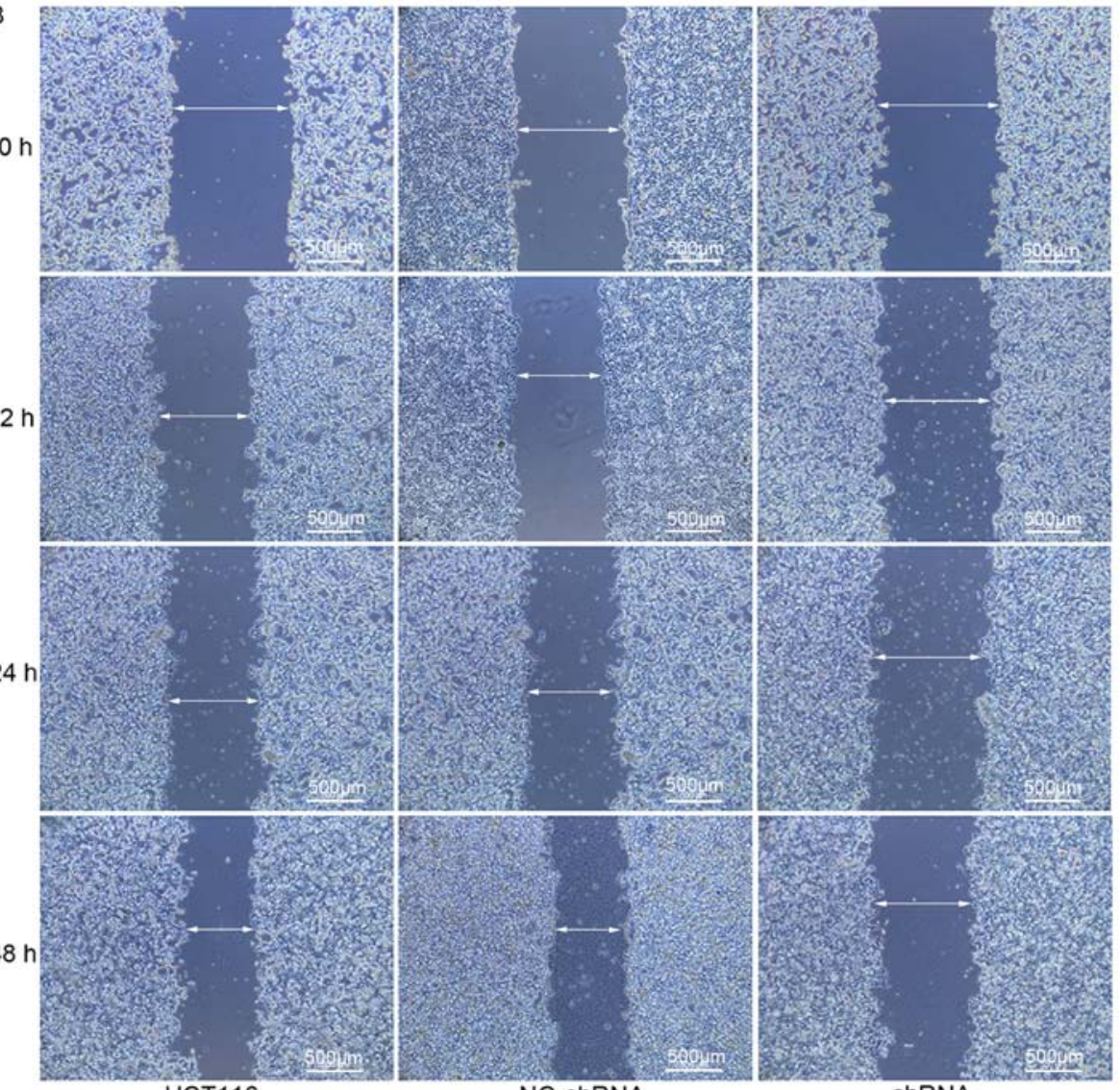

HCT116

NC-ShRNA

ShRNA

Figure 6. Knockdown of HER4 inhibits the migration of CRC cells. (A) Quantification of wound healing area. (B) Images were obtained at $0,12,24$ and $48 \mathrm{~h}$. Magnification, $x 5$. Wound healing area was measured by calculating the wound area in each period. the percentage of wound healing area=[1-(wound area at $\mathrm{Tt}$ /wound area at T0)] x100; $\mathrm{P}<0.05$ vs. the control group. CRC, colorectal cancer; shRNA, short hairpin RNA; nc-shRNA, negative control short hairpin RNA. 

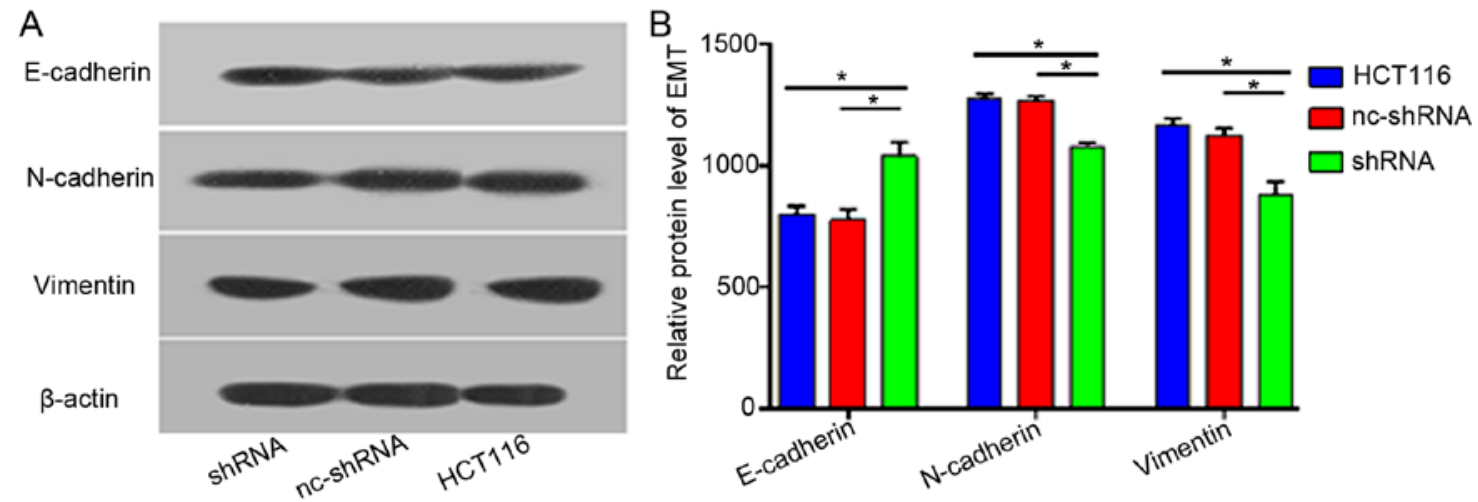

Figure 7. Knockdown of HER4 inhibits EMT in colorectal cancer cells. Western blotting results showed that knockdown of HER4 inhibited EMT-associated factors. (A) Representative images. (B) Quantification. E-cadherin is a typical marker of epithelial cells, N-cadherin and Vimentin are markers of mesenchymal cells. "P<0.05. shRNA, short hairpin RNA; nc-shRNA, negative control short hairpin RNA; EMT, epithelial-mesenchymal transition.

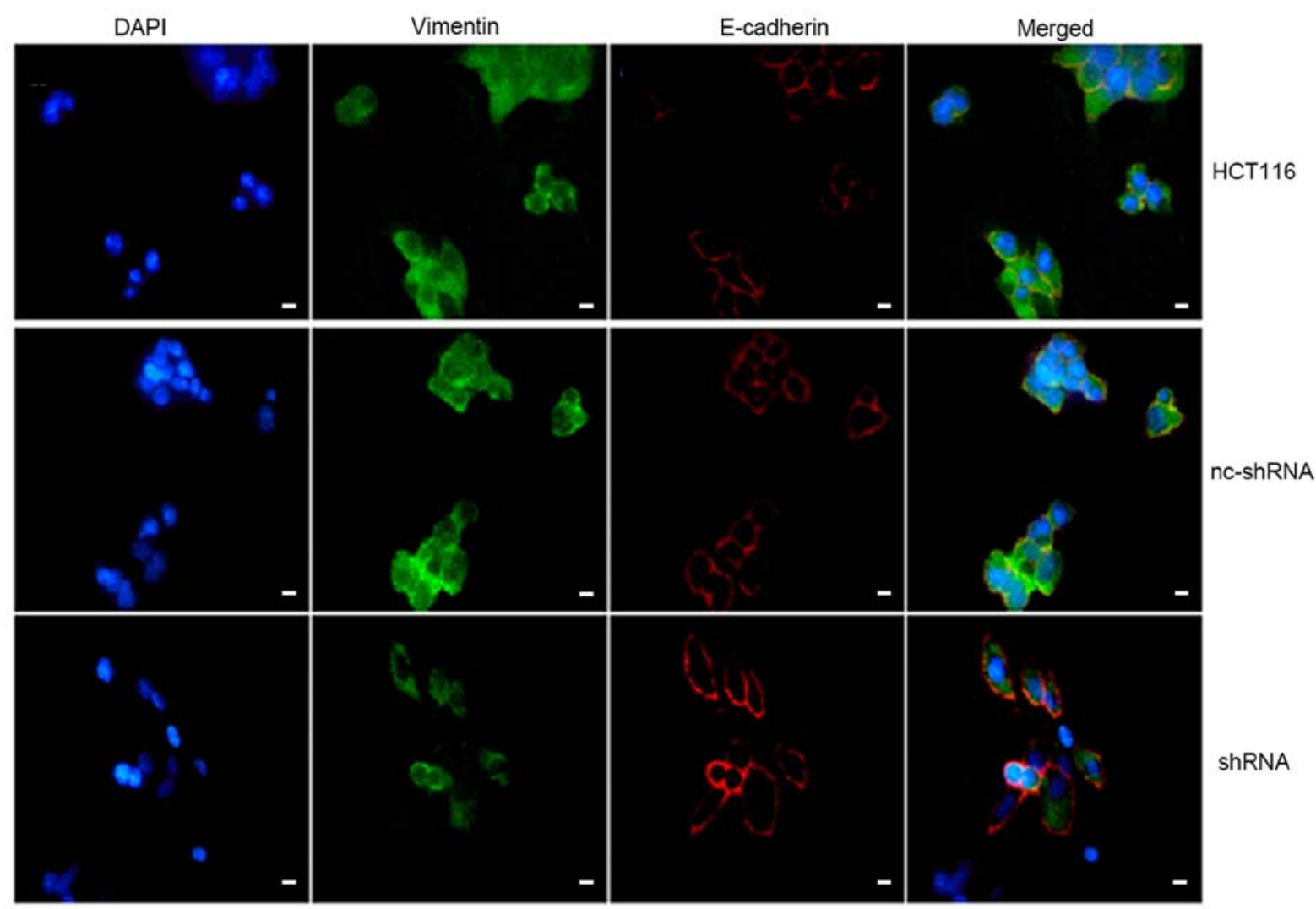

Figure 8. Immunofluorescence double staining of vimentin and E-cadherin in colorectal cancer cells. Knockdown of HER4 inhibited epithelial-mesenchymal transition. Cells are stained by DAPI in blue, by vimentin in green and by E-cadherin in red. Scale bar, $50 \mu \mathrm{m}$. shRNA, short hairpin RNA; nc-shRNA, negative control short hairpin RNA.

consistent with the present results. Therefore, further experimentation is required to test and validate the relationship among HER family members in CRC. The present results revealed that HER4 expression indicated an unfavorable prognosis in patients with CRC, and the positive rate of HER4 expression was higher in cases with lymph node metastasis. Recent studies showed that HER family members play a negative role in tumorigenesis $(29,30)$. Kountourakis et al (23) suggested that positive HER4 expression on membranes could promote lymph node metastasis. Wang et al (31) found that upregulation of HER4 indicated a poorer prognosis and higher risk for recurrence in osteosarcoma. The present results showed that the 5-year survival rate for patients with positive HER4 expression was $64.3 \%$, while that for patients with negative HER4 expression was $85.5 \%$; therefore, HER4 expression could indicate an unfavorable prognosis in patients with CRC. Furthermore, high expression of HER4 increased the risk of lymph node metastasis. However, the underlying mechanism remains unclear.

The present study found that HER4 can be found in cell membrane, cytoplasm and nucleus of CRC, which is consistent with previous results from Yun et al (32). Baiocchi et al (22) 
found that HER4 can be expressed in cytoplasm and cell membrane of cancer cells. The discrepancy in results between the studies could be due to the use of different antibodies, the present study used anti-c-HER4 antibody (cat. no. ARG52859, arigo Biolaboratories Corp.), Yun et al (32) used HER4 (Thermo Fisher Scientific, Inc.), while Baiocchi et al (22) used ErbB4 from Lab Vision Corp. (cat. no. RB-9045). Furthermore, the expression of different HER4 isoforms (CYT1 and CYT2) could impact HER4 expression localization, as the CYT2 isoform can enter the nucleus easily, but the CYT1 isoform cannot translocate into the nucleus (32).

Metastasis is a critical factor in the prognosis of patients with tumors (33). In osteosarcoma, knockdown of HER4 inhibits proliferation and tumorigenesis, induces apoptosis both in vitro and in vivo, and enhances the sensitivity to the chemotherapeutics methotrexate and doxorubicin (11). The present results showed that silencing of HER4 expression decreased proliferation and increased apoptosis of HCT116 cells, indicating that HER4 may be a valuable biomarker and potential target for CRC therapy. Therefore, HER4 may function as a carcinogenic factor in tumorigenesis.

EMT is a key step in tumor metastasis $(13,14,34)$. Previous studies have reported that microRNA-551b binds to the 3' untranslated region of HER4 and inhibits HER4 expression, reduces EMT and decreases distal metastasis in gastric cancer (35). A previous study has also confirmed that activated fatty acid synthase increases the expression of HER1, HER2 and HER4, and promotes EMT, resulting in invasion and migration induction in breast cancer (36). The present results were consistent with those of previous studies $(35,36)$. In the present study, E-cadherin expression was upregulated after knockdown of HER4, while N-cadherin and vimentin expression levels were downregulated, suggesting that HER4 expression promotes the metastasis of CRC through EMT. However, this mechanism has not yet been fully understood and requires further analysis.

The main limitation of the present study was the small clinical sample size. In addition, no relationship between the survival of patients and the expression of EGFR, HER2 and HER3 was detected. Positive HER4 expression was found in tissues of patients with CRC using IHC; however, the present study did not describe the results of the immunostaining in detail. Furthermore, the metastasis-free survival time was not recorded, thus the relationship between HER4 expression and metastasis-free survival was not examined.

With regards to the techniques used in the present study, no MTT assay was performed to detect cell proliferation. Furthermore, cells used in the wound healing assay were supplemented with $2 \%$ FBS rather than being serum-starved, and the wound healing assay was used solely to detect cell migration. In addition, the present study only investigated the effects of positive HER4 expression on cell proliferation, apoptosis, adhesion and EMT in CRC cells, but did not evaluate metastasis.

In conclusion, positive expression of HER4 was found to be associated with lymph node metastasis and could indicate an unfavorable prognosis in patients with CRC. HER4 knockdown may inhibit the growth, survival and migration of CRC cells through EMT. Therefore, the modulation of HER4 expression may be an effective therapeutic strategy for patients with CRC.

\section{Acknowledgements}

Not applicable.

\section{Funding}

This study was supported by the Natural Science Foundation of Hebei Province of China (grant. no. H2016307010).

\section{Availability of data and materials}

The datasets used and/or analyzed during the current study are available from the corresponding author on reasonable request.

\section{Authors' contributions}

$\mathrm{XJ}, \mathrm{HW}$ and $\mathrm{YJ}$ conceived and designed the experiments. XJ, JY, LG and YG conducted experiments. XJ, BW, ZL, WZ, LG and YJ performed data analysis and wrote the paper. All authors discussed the results and commented on the manuscript.

\section{Ethics approval and consent to participate}

All procedures in studies involving human participants were performed in accordance with the ethical standards of the institutional research committee (the Fourth Hospital of Hebei Medical University) and with the 1964 Helsinki Declaration and its later amendments or comparable ethical standards. The participants signed an extensive informed consent form after being informed about the benefits and risks of the procedure in the present study.

\section{Patients consent for publication}

Not applicable.

\section{Competing interests}

The authors declare that they have no competing interests.

\section{References}

1. Torre LA, Bray F, Siegel RL, Ferlay J, Lortet-Tieulent J and Jemal A: Global cancer statistics, 2012. CA Cancer J Clin 65: 87-108, 2015.

2. Sung JJ, Lau JY, Goh KL and Leung WK; Asia Pacific Working Group on Colorectal Cancer: Increasing incidence of colorectal cancer in Asia: Implications for screening. Lancet Oncol 6: 871-876, 2005.

3. Dawson H and Lugli A: Molecular and pathogenetic aspects of tumor budding in colorectal cancer. Front Med (Lausanne) 2: 11, 2015.

4. Roskoski R: The ErbB/HER family of protein-tyrosine kinases and cancer. Pharmacol Res 79: 34-74, 2014.

5. Rego RL, Foster NR, Smyrk TC, Le M, O'Connell MJ, Sargent DJ, Windschitl $\mathrm{H}$ and Sinicrope FA: Prognostic effect of activated EGFR expression in human colon carcinomas: Comparison with EGFR status. Br J Cancer 102: 165-172, 2010.

6. Kapitanović S, Radosević S, Kapitanović M, Andelinović S, Ferencić Z, Tavassoli M, Primorać D, Sonicki Z, Spaventi S, Pavelic K and Spaventi R: The expression of p185(HER-2/neu) correlates with the stage of disease and survival in colorectal cancer. Gastroenterology 112: 1103-1113, 1997. 
7. Mitsui K, Yonezawa M, Tatsuguchi A, Shinji S, Gudis K, Tanaka S, Fujimori S and Sakamoto C: Localization of phosphorylated ErbB1-4 and heregulin in colorectal cancer. BMC Cancer 14: 863, 2014.

8. Machleidt A, Buchholz S, Diermeier-Daucher S, Zeman F, Ortmann O and Brockhoff G: The prognostic value of Her4 receptor isoform expression in triple-negative and Her2 positive breast cancer patients. BMC Cancer 13: 437, 2013.

9. Göthlin EA, Tina E, Wegman P, Stål O, Fransén K, Fornander T and Wingren S: HER4 tumor expression in breast cancer patients randomized to treatment with or without tamoxifen. Int J Oncol 47: 1311-1120, 2015.

10. Nielsen TO, Poulsen SS, Journe F, Ghanem G and Sorensen BS: HER4 and its cytoplasmic isoforms are associated with progression-free survival of malignant melanoma. Melanoma Res 24 88-91, 2014.

11. Wang H, Sun W, Sun M, Fu Z, Zhou C, Wang C, Zuo D, Zhou Z, Wang $\mathrm{G}$, Zhang $\mathrm{T}$, et al: HER4 promotes cell survival and chemoresistance in osteosarcoma via interaction with NDRG1. Biochim Biophys Acta Mol Basis Dis 1864: 1839-1849, 2018

12. Guo Y, Duan Z, Jia Y, Ren C, Lv J, Guo P, Zhao W, Wang B, Zhang S, Li Y and Li Z: HER4 isoform CYT2 and its ligand NRG1III are expressed at high levels in human colorectal cancer. Oncol Lett 15: 6629-6635, 2018.

13. Thiery JP and Sleeman JP: Complex networks orchestrate epithelial-mesenchymal transitions. Nat Rev Mol Cell Biol 7: 131-142, 2006.

14. Savagner P, Boyer B, Valles AM, Jouanneau J and Thiery JP: Modulations of the epithelial phenotype during embryogenesis and cancer progression. Cancer Treat Res 71: 229-249, 1994.

15. Tam WL and Weinberg RA: The epigenetics of epithelialmesenchymal plasticity in cancer. Nat Med 19: 1438-1449, 2013.

16. $\mathrm{Vu} \mathrm{T}$ and Datta PK: Regulation of EMT in colorectal cancer: A culprit in metastasis. Cancers (Basel) 9: pii: E171, 2017.

17. Jacobs TW, Gown AM, Yaziji H, Barnes MJ and Schnitt SJ: Specificity of HercepTest in determining HER-2/neu status of breast cancers using the United States Food and Drug Administration-approved scoring system. J Clin Oncol 17: 1983-1987, 1999.

18. Williams CS, Bernard JK, Demory Beckler M, Almohazey D, Washington MK, Smith JJ and Frey MR: ERBB4 is over-expressed in human colon cancer and enhances cellular transformation. Carcinogenesis 36: 710-718, 2015.

19. Huang KK, Ramnarayanan K, Zhu F, Srivastava S, Xu C Tan ALK, Lee M, Tay S, Das K, Xing M, et al: Genomic and epigenomic profiling of high-risk intestinal metaplasia reveals molecular determinants of progression to gastric cancer. Cancer Cell 33: 137-150.e5, 2018

20. Tiwari A, Mukherjee B, Hassan MK, Pattanaik N, Jaiswal AM and Dixit M: Reduced FRG1 expression promotes prostate cancer progression and affects prostate cancer cell migration and invasion. BMC Cancer 19: 346, 2019.

21. Ljuslinder I, Malmer B, Isaksson-Mettävainio M, Oberg A, Henriksson R, Stenling R and Palmqvist R: ErbB 1-4 expression alterations in primary colorectal cancers and their corresponding metastases. Anticancer Res 29: 1489-1494, 2009.

22. Baiocchi G, Lopes A, Coudry RA, Rossi BM, Soares FA, Aguiar S, Guimarães GC, Ferreira FO and Nakagawa WT: ErbB family immunohistochemical expression in colorectal cancer patients with higher risk of recurrence after radical surgery. Int J Colorectal Dis 24: 1059-1068, 2009.
23. Kountourakis P, Pavlakis K, Psyrri A, Rontogianni D, Xiros N, Patsouris E, Pectasides D and Economopoulos T: Prognostic significance of HER3 and HER4 protein expression in colorectal adenocarcinomas. BMC Cancer 6: 46, 2006.

24. Huang F, Shi Q, Li Y, Xu L, Xu C, Chen F, Wang H, Liao H, Chang Z, Liu F, et al: HER2/EGFR-AKT signaling switches TGF- $\beta$ from inhibiting cell proliferation to promoting cell migration in breast cancer. Cancer Res 78: 6073-6085, 2018.

25. Siddiqa A, Long LM, Li L, Marciniak RA and Kazhdan I: Expression of HER-2 in MCF-7 breast cancer cells modulates anti-apoptotic proteins Survivin and Bcl-2 via the extracellular signal-related kinase (ERK) and phosphoinositide-3 kinase (PI3K) signalling pathways. BMC Cancer 8: 129, 2008.

26. Béguelin W, Díaz Flaqué MC, Proietti CJ, Cayrol F, Rivas MA, Tkach M, Rosemblit C, Tocci JM, Charreau EH, Schillaci R and Elizalde PV: Progesterone receptor induces ErbB-2 nuclear translocation to promote breast cancer growth via a novel transcriptional effect: ErbB-2 function as a coactivator of Stat3. Mol Cell Biol 30: 5456-5472, 2010.

27. Lo HW: Targeting Ras-RAF-ERK and its interactive pathways as a novel therapy for malignant gliomas. Curr Cancer Drug Targets 10: 840-848, 2010.

28. Lee JC, Wang ST, Chow NH and Yang HB: Investigation of the prognostic value of coexpressed erbB family members for the survival of colorectal cancer patients after curative surgery. Eur J Cancer 38: 1065-1071, 2002.

29. Donoghue JF, Kerr LT, Alexander NW, Greenall SA, Longano AB, Gottardo NG, Wang R, Tabar V, Adams TE, Mischel PS and Johns TG: Activation of ERBB4 in glioblastoma can contribute to increased tumorigenicity and influence therapeutic response. Cancers (Basel) 10: pii: E243, 2018

30. Shi J, Li F, Yao X, Mou T, Xu Z, Han Z, Chen S, Li W, Yu J, Qi X, et al: The HER4-YAP1 axis promotes trastuzumab resistance in HER2-positive gastric cancer by inducing epithelial and mesenchymal transition. Oncogene 37: 3022-3038, 2018.

31. Wang W, Zhao HF, Yao TF and Gong H: Advanced development of ErbB family-targeted therapies in osteosarcoma treatment. Invest New Drugs 37: 175-183, 2019.

32. Yun S, Kwak Y, Nam SK, Seo AN, Oh HK, Kim DW, Kang SB and Lee HS: Ligand-independent epidermal growth factor receptor overexpression correlates with poor prognosis in colorectal cancer. Cancer Res Treat 50: 1351-1361, 2018.

33. De Craene B and Berx G: Regulatory networks defining EMT during cancer initiation and progression. Nat Rev Cancer 13: 97-110, 2013

34. Sulaiman A, Li L and Wang L: E-cadherin adhesion-mediated Wnt activation for mesoderm specification in human embryonic stem cells needs a soft mattress. Stem Cell Investig 3: 77, 2016.

35. Song G, Zhang H, Chen C, Gong L, Chen B, Zhao S, Shi J, Xu J and Ye Z: MiR-551b regulates epithelial-mesenchymal transition and metastasis of gastric cancer by inhibiting ERBB4 expression. Oncotarget 8: 45725-45735, 2017.

36. Chen T, Zhou L, Li H, Tian Y, Li J, Dong L, Zhao Y and Wei D: Fatty acid synthase affects expression of ErbB receptors in epithelial to mesenchymal transition of breast cancer cells and invasive ductal carcinoma. Oncol Lett 14: 5934-5946, 2017.

This work is licensed under a Creative Commons

Attribution-NonCommercial-NoDerivatives 4.0

International (CC BY-NC-ND 4.0) License. 